\title{
CORRECTIONS
}

\section{The importance of statistical power}

The sixth paragraph of the answers section in this Endgames quiz (BMJ 2013;347:f6282, doi:10.1136/bmj.f6282) contains an error. In the third sentence the mistaken use of the word "inadequate," which created a double negative, should be replaced by "adequate" so that the sentence fully reads: "If the sample size was too small it would not have adequate power and might fail to detect the smallest effect of clinical interest, if it existed in the population."

Cite this as: BMJ 2013;347:f6441

๑ BMJ Publishing Group Ltd 2013 